\title{
Análisis de la capacidad de innovación regional: el caso español
}

\author{
López-Fernández, María Concepción* \\ Serrano-Bedia, Ana María** \\ García-Piqueres, Gema***
}

\section{Resumen}

El objetivo del presente trabajo es estudiar los determinantes de la capacidad de innovación en las regiones españolas. Para ello se utiliza el modelo desarrollado por Furman, Porter y Stern (2002) aplicándolo a nivel regional, lo que permite comprobar si dicho modelo es pertinente para el estudio de los determinantes de la capacidad de innovación regional, configurándose así como un segundo objetivo del trabajo. Para alcanzar dichos objetivos se llevó a cabo un estudio econométrico basado en la metodología de datos de panel, con una estimación de efectos aleatorios, a partir de datos tomados del Instituto Nacional de Estadística (INE). Los resultados del estudio empírico ponen de manifiesto la importancia de la infraestructura común de la innovación y la calidad de los vínculos como principales factores determinantes de la capacidad de innovación regional. Por tanto, los resultados estadísticos sugieren que en el caso español, las dimensiones infraestructura común y calidad de los vínculos juegan un papel fundamental para el desarrollo de las capacidades de innovación de las regiones, mientras que la importancia de la actividad empresarial como motor de generación de innovaciones parece más limitada en el caso de las regiones españolas.

Palabras clave: Capacidad de innovación, España, infraestructura común para la innovación, entorno específico de innovación de los clusters, calidad de los vínculos, datos de panel.

Recibido: 16-11-10. Aceptado: 23-05-11

* Profesora Titular del Departamento de Administración de Empresas de la Universidad de Cantabria. E-mail: lopezm@unican.es.

** Profesora Titular del Departamento de Administración de Empresas de la Universidad de Cantabria. E-mail: serranoa@unican.es.

*** Ayudante en el Departamento de Administración de Empresas de la Universidad de Cantabria. E-mail: garciapg@unican.es. 
Análisis de la capacidad de innovación regional: el caso español

López-Fernández, María C.; Serrano-Bedia, Ana M. y García-Piqueres, Gema

\title{
Analysis of Regional Innovative Capacity: The Spanish Case
}

\begin{abstract}
The paper aims to study determinants for innovative capacity in the Spanish regions. To achieve this objective, the model developed by Furman, Porter and Stern (2002) was applied at a regional level, making it possible to test whether or not this model is applicable at the regional level; this constitutes a second objective of this work. To achieve these goals, an econometric study based on panel data methodology was carried out, estimating random effects, based on data from the National Statistics Institute. Empirical results reveal the importance of common innovation infrastructure and the quality of linkages as principal determinants for regional innovative capacity. Therefore, statistical results suggest that for the Spanish case, the dimensions of common infrastructure and the quality of linkages play a fundamental role in developing capacities for innovation in the regions, while the importance of entrepreneurial activity as a motor for generating innovations seems to be more limited in Spanish regions.
\end{abstract}

Key words: Innovative capacity, Spanish regions, common infrastructure for innovation, clusterspecific innovation environment, quality of linkages, panel data.

\section{Introducción}

En la economía moderna, la innovación es considerada como un factor clave por cuanto que en todas las partes de la economía pueden identificarse procesos de producción de conocimiento que favorecen la generación de innovaciones (Batlle y Osorio, 2009). Por lo tanto, se puede argumentar que la competitividad de las empresas y de la economía en su conjunto va a depender en gran medida de su capacidad de innovación (Porter, 1990; Ojeda, 2007; Estrada et al., 2009).

La importancia de la innovación en términos de crecimiento económico ha sido estudiada en la literatura y los economistas han adoptado diferentes enfoques para el análisis de esta relación (Fagerberg et al., 2006). Debido a la mencionada importancia de la innovación para el crecimiento económico, que ha sido apoyada tanto desde un punto de vista teóri- co como empírico, la literatura ha abordado el estudio de los factores que determinan la capacidad de innovación (Varsakelis, 2006). Tomando este supuesto como punto de partida, y motivados asimismo por las diferencias en términos de capacidad de innovación entre países, Furman, Porter y Stern (2002) desarrollaron un modelo basado en el concepto de capacidad de innovación nacional, entendiéndose esta última como la habilidad de un país para producir y comercializar un flujo determinado de innovación a lo largo del tiempo. Este modelo investiga el conjunto de fuentes de innovación a nivel país apoyándose en tres enfoques teóricos relacionados con las fuentes de innovación: la Teoría del Crecimiento Endógeno (Romer, 1986), la Teoría de Clusters de la Ventaja Competitiva Nacional (Porter, 1990) y la Teoría de los Sistemas Nacionales de Innovación (Nelson, 1993; Dosi, 1988; Edquist, 1997). 
Paralelamente al desarrollo del análisis de los procesos de innovación a nivel nacional, la importancia de la innovación a nivel regional ha sido puesta de manifiesto en las economías industrializadas desde mediados de los años 70 (Park y Lee, 2005), en gran parte fruto de la existencia de políticas aplicables a nivel regional (Porter, 1990). Así mismo, en un entorno regional es más fácil conseguir apoyo para la obtención de la comunicación necesaria para el desarrollo de innovaciones gracias fundamentalmente a la proximidad (Gregersen y Johnson, 1997). Por lo tanto, la región se configura como un entorno favorable para el desarrollo de innovaciones, que en ocasiones puede ser complejo y requerir del desarrollo de acuerdos de cooperación entre diferentes agentes del entorno regional (Meyer-Kramer, 2001). Por último, y como consecuencia del proceso de regionalización y globalización, el entorno regional toma tanta relevancia como el nacional en la determinación de la capacidad de las empresas para competir globalmente (Chatterton y Goddard, 2000). En el caso de Europa, las regiones juegan un papel principal en términos de desarrollo económico (Park y Lee, 2005). En esta línea, las instituciones de la Unión Europea prestan cada vez más atención a los procesos de descentralización regional, hablándose así de la "Europa de las regiones" (Fernández de Lucio, 2001). De manera más precisa, para países como España, un análisis a este nivel puede ser de interés debido a que las capacidades, recursos y resultados relacionados con la innovación varían de unas regiones a otras (Coronado y Acosta, 1999; Baumert y Heijs, 2002; Buesa et al.,
2006). En este sentido, España constituye un caso paradigmático de estudio a nivel regional como consecuencia de su estructura territorial, que se configura en torno a 17 Comunidades Autónomas que poseen competencias a niveles políticos, administrativos y económicos dentro de los que se encuentran los relativos a la innovación y el desarrollo tecnológico (Fernández de Lucio et al., 2007). Dentro del contexto europeo, España se encuadra, por un lado, en el grupo de regiones con poderes avanzados (Wiehler y Stumm, 1995) y un grado sustancial de autonomía (Fernández de Lucio et al., 2007), frente a otros países cuyas regiones presentan niveles de descentralización mucho menores como Francia, Portugal o Reino Unido (regiones con poderes limitados). Por otro lado, las regiones españolas son clasificadas por la Comisión Europea (2001) dentro de la categoría de "Regiones Periféricas". Una región no es periférica exclusivamente en sentido geográfico, sino que las regiones periféricas son también aquellas que parten de condiciones de desventaja para su territorio en términos de menor equipamiento, menor base industrial, débil red empresarial y de negocios o agentes con escasa cultura innovadora (González Ramos y González de la Fe, 2009).

Respecto a esta última tipología regional (periférica), su análisis resulta de interés dado que la mayor parte de la literatura previa se ha centrado en las regiones más exitosas en términos de innovación como el caso japonés, Silicon Valley, Manchester o Lyon, obteniéndose resultados difícilmente extrapolables para el caso de regiones periféricas (González Ramos y González de la Fe, 2009). De 
Análisis de la capacidad de innovación regional: el caso español

López-Fernández, María C.; Serrano-Bedia, Ana M. y García-Piqueres, Gema

este modo, el estudio de regiones periféricas como las españolas, puede arrojar cierta luz sobre este tema, permitiendo así mismo obtener conclusiones más aplicables para otros contextos regionales periféricos tanto dentro como fuera de la Unión Europea.

Asumiendo la importancia del análisis a nivel regional para el caso español, el objetivo del presente trabajo es estudiar de los determinantes de la capacidad de innovación de las regiones españolas. Para ello se va a utilizar el modelo desarrollado por Furman, Porter y Stern (2002) aplicándolo a nivel regional. Por lo tanto, son dos los principales aportes que de este trabajo se derivan para nuestro estudio: el primero se basa en que nuestro estudio va a permitir conocer los factores impulsores de la capacidad de innovación regional para al caso español; el segundo de ellos, es que con el estudio vamos a poder comprobar si el modelo de Furman, Porter y Stern (2002) es pertinente para el estudio de los determinantes de la capacidad de innovación regional. Para alcanzar los objetivos del trabajo, el estudio empírico desarrolla los siguientes análisis. En primer lugar, el estudio presenta las frecuencias y correlaciones entre las diferentes variables tomadas de la revisión de la literatura como determinantes de la capacidad de innovación. Además, se analiza si los valores de las diferentes variables varían significativamente de unas regiones a otras. En segundo lugar, se aborda el estudio de los determinantes de la capacidad de innovación regional mediante análisis de regresión. Dada la tipología de datos que se utilizan en el estudio, concretamente encuestas realizadas por el Instituto $\mathrm{Na}$ cional de Estadística (INE) que proporcio- nan información de las variables para diferentes años (2001 a 2005), la aplicación de la metodología de datos de panel es posible.

Con el fin de desarrollar los planteamientos anteriores, el trabajo se ha estructurado como sigue. La segunda sección presenta el desarrollo del trabajo en el que se recoge la literatura acerca de los determinantes de la capacidad de innovación a nivel geográfico, así como el estudio realizado para el caso de las regiones españolas. Y en la tercera y última sección, se desarrollan las conclusiones, limitaciones e implicaciones de la investigación.

\section{Determinantes de la capacidad de innovación regional}

Puesto que el presente trabajo basa su análisis en el modelo desarrollado por Furman, Porter y Stern (2002) para el estudio de los determinantes de la capacidad de innovación nacional, a continuación se sistematiza dicho modelo.

\subsection{El modelo de Furman, Porter y Stern (2002)}

Se fundamenta en un enfoque integrador, a través del cual se identifican el conjunto de dimensiones principales que determinan la capacidad de innovación nacional. En concreto, los autores identifican tres dimensiones (Figura 1):

1. La infraestructura común de la innovación.

2. El entorno de innovación específico de los clusters.

3. La calidad de los vínculos entre las dos dimensiones anteriores. 
Figura 1

\section{Modelo de la Capacidad de Innovación Nacional}

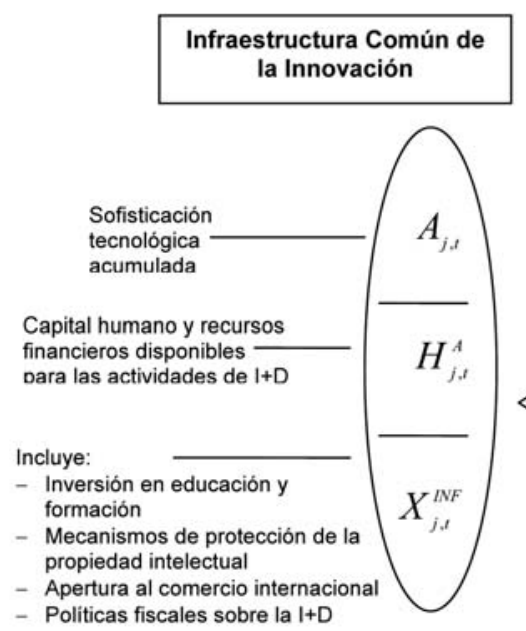

- Politicas fiscales sobre la I+D

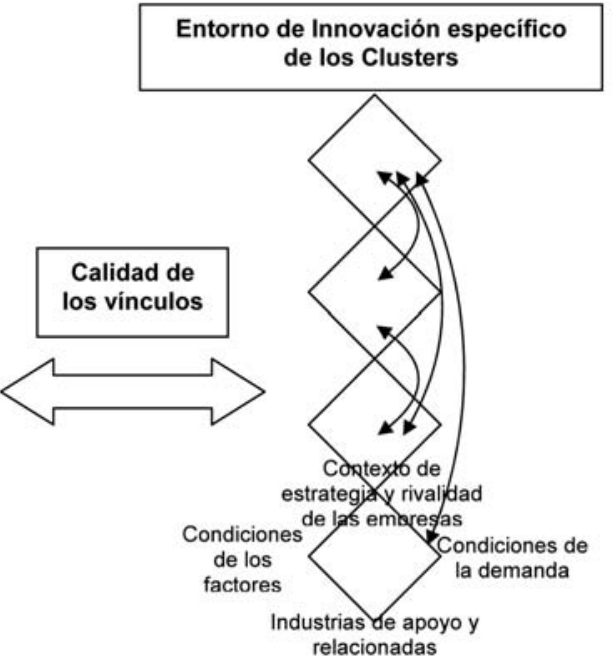

dustrias de apoyo y

Fuente: Furman, Porter y Stern (2002).

Para la construcción del modelo los autores se apoyaron principalmente en tres enfoques teóricos:

1. La Teoría del Crecimiento Endógeno impulsada por Romer (1986).

2. La Teoría basada en clusters de la Ventaja Competitiva Nacional (Porter, 1990).

3. La Teoría de los Sistemas Nacionales de Innovación (Freeman, 1987).

En este sentido, la dimensión "infraestructura común de la innovación" incluye diversos elementos procedentes de los enfoques de la Teoría del Crecimiento Endógeno y los Sistemas Nacionales de Innovación. Por lo que se refiere al "entorno específico de los cluster", su fundamentación se basa principalmente en la teoría de los clusters de la ventaja competitiva industrial nacional de Porter y en el enfoque de los Sistemas Nacionales de
Innovación. Por último, en cuanto a "la calidad de los vínculos”, esta dimensión parece más asociada a los enfoques de la teoría de los Clusters de la Ventaja Competitiva Industrial Nacional y de los Sistemas Nacionales de Innovación.

En ese sentido, es pertinente exponer de manera detallada cada una de las tres dimensiones identificadas en el modelo, así como la evidencia empírica obtenida en los trabajos que han usado este enfoque y cuya información se recoge en los Cuadros 1 y 2. El primero de éstos presenta la muestra de países objeto de estudio en cada uno de dichos trabajos, así como el periodo temporal de los mismos. El Cuadro 2, por su parte, resume los trabajos que han estudiado empíricamente los determinantes de la capacidad de innovación nacional, basándose principalmente en el modelo de Furman, Por- 
Análisis de la capacidad de innovación regional: el caso español

López-Fernández, María C.; Serrano-Bedia, Ana M. y García-Piqueres, Gema
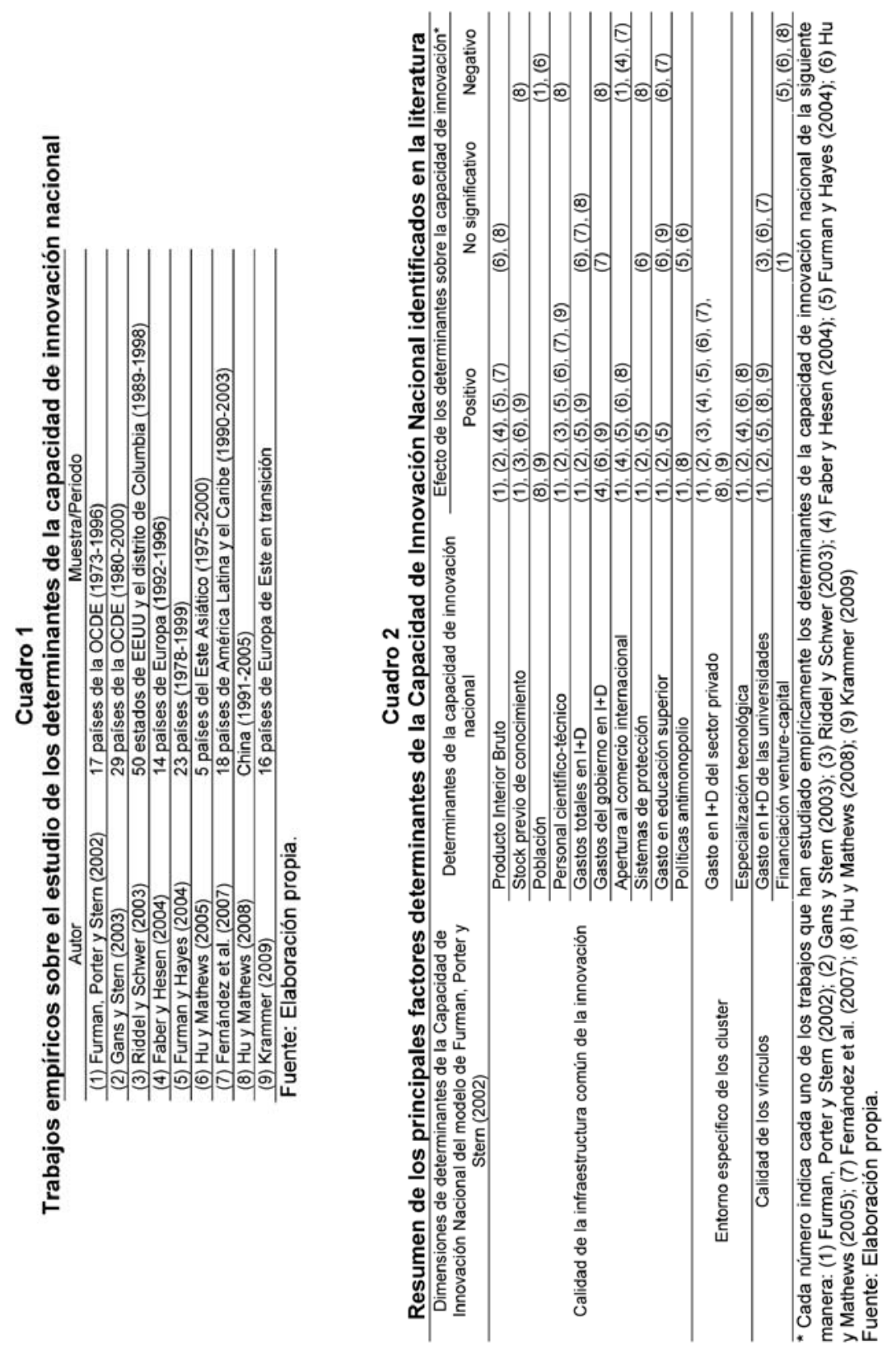
ter y Stern (2002). De manera más precisa, el cuadro resume las variables analizadas en cada uno de los trabajos, así como el efecto de éstas sobre la capacidad de innovación nacional para cada caso.

1. Infraestructura común de innovación

Esta primera dimensión de determinantes de la capacidad de innovación nacional va a estar formada por tres factores relativos a las inversiones que apoyan las actividades innovadoras: el nivel agregado de sofisticación tecnológica de una economía $\left(A_{t}\right)$, la masa disponible de personal científico-técnico cualificado dedicada a la I+D $\left(H_{A, t}\right)$ y un tercer grupo de variables relacionado con inversiones nacionales y decisiones de políticas de innovación $\left(X_{I N F, t}\right)$.

Comenzando con los factores $A_{t} y$ $\mathrm{H}_{A, t}$ su identificación se basa principalmente en el modelo de crecimiento endógeno formulado por Romer (1986; 1990), según el que la generación de nuevos conocimientos a nivel nacional va a depender no sólo del esfuerzo en I+D llevado a cabo, sino también del stock de conocimientos acumulados o previos al período de estudio. Según este modelo la tasa de cambio tecnológico es endógena de dos maneras diferentes; en primer lugar, el porcentaje de la economía dedicado a generar ideas en los sectores es una función de la I+D del mercado de trabajo; en segundo lugar, la productividad de la generación de nuevas ideas es sensible al stock de ideas generado en el pasado. Este planteamiento se conoce como el enfoque de la Función de Producción de Ideas (Knowledge Production FunctionKPF-) que relaciona inputs y outputs liga- dos a los procesos de innovación (Krammer, 2009).

La función que se deriva de este enfoque es:

$$
A_{t}=\delta \mathrm{H}_{\mathrm{At},}^{\lambda} A_{t}^{\varphi}
$$

donde:

$A_{t}$ es el flujo de nuevos conocimientos, $H_{A, t}^{\lambda}$ es el personal dedicado a I+D y, $A_{t}^{\varphi}$ es el stock de conocimientos acumulados.

Empíricamente, diversos autores han estudiado el efecto de estos dos factores sobre la capacidad de innovación nacional (Cuadro 2). Por lo que al stock de conocimiento previo o sofisticación tecnológica se refiere $\left(A_{j, t}^{\phi}\right)$ los diferentes estudios toman como indicadores el PIB per cápita o el stock previo de patentes. Para ambas variables se tiene un efecto mayoritariamente positivo de sobre la capacidad de innovación nacional, tal y como se desprende del Cuadro 2. Respecto del capital humano y financiero $\left(H_{j, t}^{\mathrm{A} \lambda}\right)$, los indicadores utilizados son la población, el personal científico-técnico y los gastos en I+D. En primer lugar, en cuanto al indicador de la población la evidencia empírica no es concluyente. Por un lado, si bien diferentes trabajos encuentran un efecto positivo de la población sobre la capacidad de innovación nacional (Hu y Mathews, 2008; Krammer, 2009), otros (Furman, Porter y Stern, 2002; Hu y Mathews, 2005) obtienen el resultado contrario. En segundo lugar, la evidencia empírica disponible confirma mayoritariamente un efecto positivo del personal científico-técnico y los gastos en I+D sobre la capacidad de innovación nacional (Cuadro 2). 
Análisis de la capacidad de innovación regional: el caso español López-Fernández, María C.; Serrano-Bedia, Ana M. y García-Piqueres, Gema

Desde el punto de vista del enfoque de Sistemas Nacionales de Innovación ${ }^{1}$, Furman, Porter y Stern (2002) incorporan a su modelo un tercer grupo de factores relacionado con inversiones nacionales y decisiones de políticas de innovación $\left(\left(X_{I N F, t}\right)\right.$. El enfoque de los Sistemas Nacionales de Innovación se centra en la descripción de la organización y tipos de actividad que influyen en el comportamiento innovador de los países, subrayando el papel de las políticas gubernamentales e instituciones específicas. Dentro de este enfoque el papel de los factores relacionados con inversiones nacionales y políticas de innovación ha sido puesto de manifiesto por diversos autores (Lundvall, 1992; Fernández de Lucio et al., 1996; COTEC, 2004; Heijs et al., 2007).

A partir de esta línea de investigación, Furman, Porter y Stern (2002) identifican el siguiente conjunto de factores que repercuten en la actividad innovadora $\left(X_{I N F, t}\right)$ : las inversiones nacionales, la inversión en educación superior, las políticas públicas relacionadas con los sistemas de patentes o leyes sobre copyright, la extensión de los créditos fiscales para la I+D, las leyes antimonopolio, la tasación de las ganancias de capital o la apertura de la economía a la competencia internacional.
Empíricamente, esta dimensión $\left(X_{j, t}^{I N F}\right)$ se ha medido a través del nivel del gasto de las administraciones públicas en I+D, la apertura al comercio internacional, los sistemas de protección de la propiedad intelectual, el porcentaje de gasto en educación superior sobre el PIB y las políticas antimonopolio. Comenzando con el gasto de las administraciones en I+D, su efecto sobre la capacidad de innovación nacional se confirma como mayoritariamente positivo en base a la evidencia empírica previa (Cuadro 2). En cuanto a la apertura internacional, la evidencia empírica no es concluyente. Por un lado su efecto positivo sobre la capacidad de innovación nacional es empíricamente confirmado en Furman, Porter y Stern (2002) (cuando se toma el PIB per capita como indicador de $A_{j, t}^{\phi}$ ), Faber y Hesen (2004) (cuando se toma el número de patentes como indicador de la capacidad de innovación nacional), Furman y Hayes (2004), Hu y Mathews (2005) y Hu y Mathews (2008). Por otro lado, se tiene un efecto negativo y significativo en Furman, Porter y Stern (2002) cuando toman el stock previo de patentes como indicador de $A_{j, t}^{\phi}$, en Faber y Hesen (2004) cuando toman el porcentaje de ventas de productos innovadores

1 Si bien Lundvall (1985) fue quien introdujo el concepto de Sistema de Innovación en términos generales sin aludir a la dimensión nacional, Freeman (1987) que fue quién usó por primera vez la expresión de "Sistemas Nacionales de Innovación" (SNI) en una obra sobre la innovación en Japón en la que el autor definía estos como "la red de instituciones, tanto en los sectores públicos como privados, cuyas actividades e interacciones inician, importan, modifican y difunden nuevas tecnologías". 
sobre el total como indicador de la capacidad de innovación nacional y en Fernández et al. (2007). Respecto de los efectos de los instrumentos de protección de la propiedad intelectual sobre la capacidad de innovación nacional la evidencia empírica disponible sugiere un efecto mayoritariamente positivo (Cuadro 2). Continuando con el porcentaje de gasto en educación superior sobre el PIB se refiere, la evidencia empírica no es concluyente, con trabajos que confirman un efecto positivo (Furman, Porter y Stern, 2002; Gans y Stern, 2003; Furman y Hayes, 2004), negativo (Fernández et al., 2007), o sin efecto (Krammer, 2009). En Hu y Mathews (2005) no se confirma ninguna relación en términos, cuando se toma el PIB per cápita como indicador de $A_{j, t}^{\phi}$, si bien cuando dichos autores toman el stock previo de patentes como indicador de $A_{j, t}^{\phi}$ confirman un efecto negativo (Cuadro 2). Y en último lugar, la evidencia empírica apunta a un efecto positivo de las políticas antimonopolio sobre la capacidad de innovación nacional (Cuadro 2). La Figura 2 resume los enfoques teóricos, las dimensiones y factores así como los principales resultados de la literatura empírica respecto de la dimensión infraestructura común de la innovación.

\section{Entorno de innovación especí- fico de los clusters \\ Esta segunda dimensión de deter-} minantes de la capacidad de innovación nacional está formada por el conjunto de factores relativos a las empresas y el entorno microeconómico que apoyan las actividades innovadoras. Para ello los autores se apoyan en los fundamentos procedentes de la Teoría basada en los clusters de la Ventaja Competitiva Nacional (Porter, 1990) y el enfoque de Sistemas Nacionales de Innovación. Desde este punto de vista Furman, Porter y Stern (2002) consideran que si bien la infraestructura común de la innovación determina el conjunto general de recursos de apoyo a la innovación disponibles en una economía son las empresas en último lugar, influenciadas por su entorno microeconómico, las que introducen y comercializan las innovaciones.

Basándose en el marco introducido por Porter (1990), denominado el "diamante", que enumeraba el conjunto de características del entorno en los clusters $^{2}$ de un país, Furman, Porter y Stern (2002) destaca cuatro elementos clave del entorno macroeconómico: la presencia de factores (inputs) especializados y de gran calidad; un contexto que fomente la inversión y la rivalidad local intensa; la presión y la percepción recogida por la demanda local sofisticada; y la presencia

2 Porter (1990) define los clusters como "concentraciones geográficas de empresas interconectadas, suministradores especializados, proveedores de servicios, empresas de sectores afines e instituciones conexas unidas por rasgos comunes y complementarias entre sí, que compiten pero también cooperan". 
Análisis de la capacidad de innovación regional: el caso español

López-Fernández, María C.; Serrano-Bedia, Ana M. y García-Piqueres, Gema

Figura 2

\section{La infraestructura común de la innovación en el modelo de Furman, Porter y Stern (2002)}

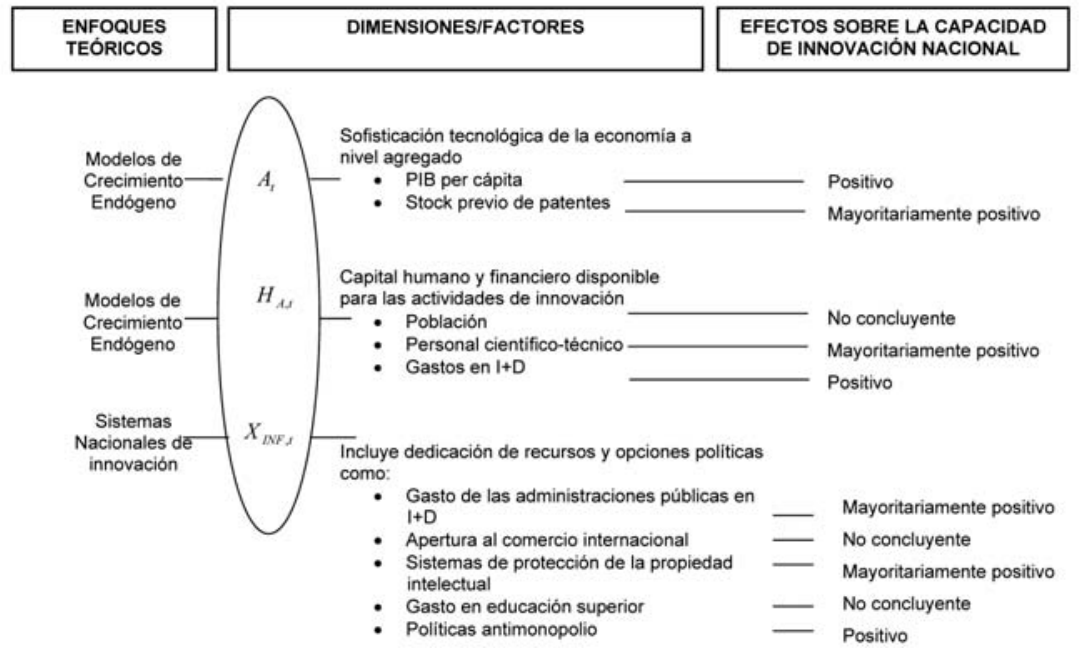

Fuente: Elaboración propia.

de un cluster de industrias relacionadas y de apoyo. Los planteamientos de Porter en este sentido señalan que, en última instancia, la competitividad de un país va a ser consecuencia de la competitividad e innovación de las empresas de ese país, que va a beneficiarse de la existencia de clusters industriales (Mitxeo et al., 2004).

Desde la perspectiva de los Sistemas Nacionales de Innovación, diversos autores han destacado la importancia de la actividad empresarial para el desarrollo de innovaciones en un país (Nelson, 1993; Fernández de Lucio et al., 1996; COTEC, 2004; Heijs et al., 2007) poniendo énfasis en el hecho de que las empresas son las instituciones que promueven la creación y difusión de conocimiento, situándose como principales fuentes de información. Los diferentes autores destacan también el papel de las empresas dentro de los Sistemas Nacionales de Innovación, a través del esfuerzo en I+D y su cultura innovadora, el tamaño de las empresas y el nivel de concentración, la estructura de los mercados, las condiciones de la demanda y la distribución sectorial.

A partir de estas líneas de investigación, Furman, Porter y Stern (2002) identifican los siguientes factores que dentro del entorno de innovación específico de los cluster repercuten en la actividad innovadora: el esfuerzo innovador de las empresas y la especialización productiva de la economía de un país. Empíricamente se han tomado como indicadores de esta dimensión el peso del sector privado en la financiación de las actividades innovadoras de una economía y la especialización del país (concentración relativa del output innovador en actividades in- 
novadoras). Para ambas variables la evidencia empírica previa sugiere un efecto positivo sobre la capacidad de innovación (Cuadro 2). La Figura 3 resume los enfoques teóricos, las dimensiones y variables así como los principales resultados de la literatura empírica respecto del entorno específico de los cluster.

\section{Calidad de los vínculos del sistema}

Por último, en base a los enfoques de la Teoría basada en los clusters de la Ventaja Competitiva Nacional y el enfoque de Sistemas Nacionales de Innovación, Furman, Porter y Stern (2002) identifican esta tercera dimensión de factores determinantes de la capacidad de innovación nacional.
Desde esta perspectiva, la relación entre la infraestructura común de la innovación y el entorno específico de los clusters es bidireccional de manera que la producción de innovaciones, condicionada por el entorno de innovación en cualquier cluster, aumentará con la potencia de la infraestructura común de innovación de la economía (Porter et al., 2001). El modelo se basa en considerar que la fuerza o calidad de los vínculos entre las dos dimensiones va a determinar el nivel de producción de innovaciones en los clusters industriales sostenidos por la infraestructura común de innovación. Por lo tanto, la interrelación entre los dos elementos se configura como uno de los principales impulsores de la capacidad de innovación.

Figura 3

\section{Entorno de innovación específico de los clusters en el modelo} de Furman, Porter y Stern (2002)

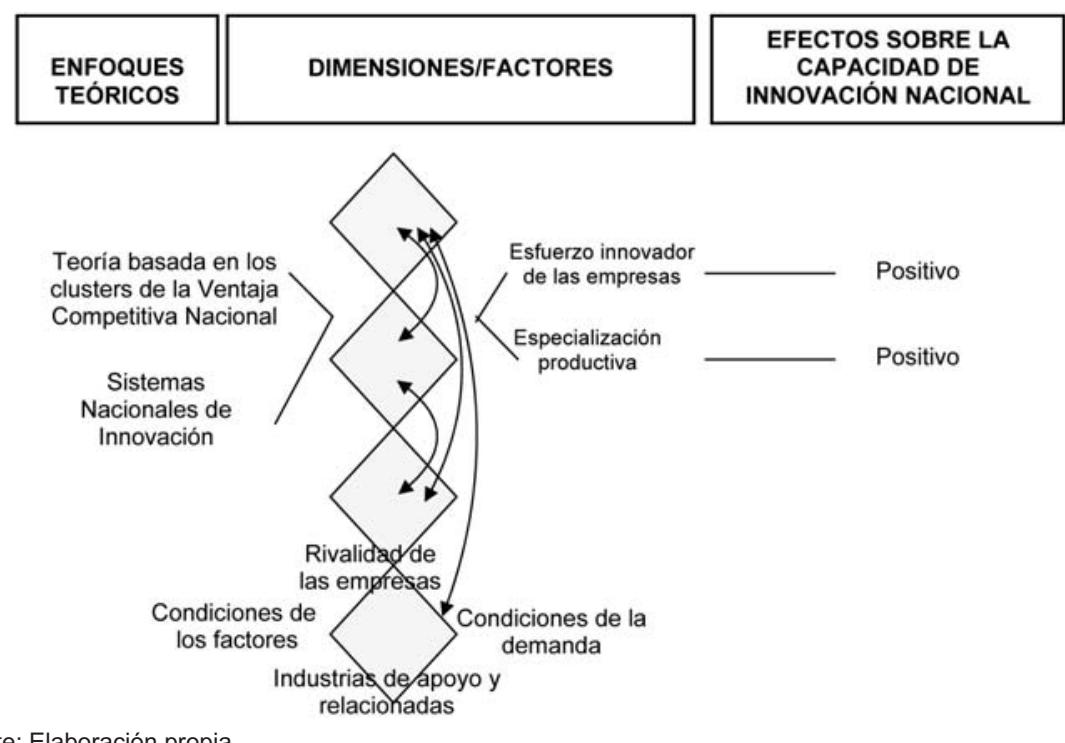

Fuente: Elaboración propia. 
Análisis de la capacidad de innovación regional: el caso español

López-Fernández, María C.; Serrano-Bedia, Ana M. y García-Piqueres, Gema

Por lo que al modelo del diamante de Porter se refiere, diversos autores han resaltado la importancia que pueden tener las interacciones entre los clusters y determinadas instituciones específicas como universidades o centros de investigación dentro de determinadas áreas geográficas (Porter, 1990; Niosi, 1991; Mowery, 1984). Continuando con la teoría sobre Sistemas Nacionales de Innovación, desde este punto de vista las interrelaciones entre diferentes agentes del sistema constituyen una de las vías más importantes para el desarrollo de innovaciones (Freeman, 1987; Lundvall, 1992; Nelson, 1993). A partir de estos enfoques Furman, Porter y Stern (2002) toman dos principales indicadores que configuran la dimensión de calidad de los vínculos. Por un lado, la tasa del gasto en I+D llevado a cabo por las universidades y por otro, la disponibilidad de apoyo para la financiación de venture-capital. En primer lugar, respecto de la inversión de las universidades en I+D, un efecto mayoritariamente positivo sobre la capacidad de innovación nacional se contrasta empíricamente (Cuadro 2). En segundo lugar, la mayo- ría de la evidencia empírica disponible apunta a un efecto negativo de la disponibilidad de apoyo financiero a la creación de venture-capital en la capacidad de innovación nacional (Cuadro 2). La Figura 4 resume los enfoques teóricos, las dimensiones y variables así como los principales resultados de la literatura empírica respecto de la dimensión calidad de los vínculos.

A modo de síntesis, cabe señalar en primer lugar que la mayor parte de trabajos que han abordado el estudio de los determinantes de la capacidad de innovación nacional se han basado fundamentalmente en el modelo desarrollado por Furman, Porter y Stern (2002), tomando las dimensiones propuestas por dichos autores como principales factores condicionantes de la capacidad de innovación. En segundo lugar, destaca que la evidencia empírica es mayoritariamente coincidente en que los factores de tales dimensiones actúan como determinantes de la capacidad de innovación, demostrando por tanto, la validez del modelo y su pertinencia para la realización de estudios empíricos. En este sentido, cabe

Figura 4

La calidad de los vínculos del sistema en el modelo de Furman, Porter y Stern (2002)

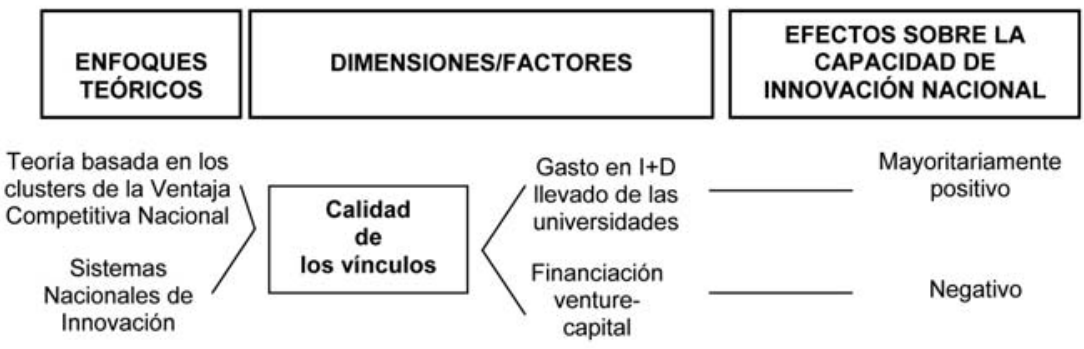

Fuente: Elaboración propia. 
destacar asimismo que dicha validez y pertinencia del modelo se ve reforzada por el hecho de que el mismo ha sido contrastado para un amplio número de países (Cuadro 1). Todas estas cuestiones proporcionan fiabilidad no sólo al propio modelo en sí mismo, sino a la fundamentación teórica y metodológica empleada para su construcción.

En base a todos estos argumentos, a continuación se desarrolla un estudio empírico para analizar los determinantes de la capacidad de innovación a nivel regional basado en el conjunto de variables identificadas en el modelo de Capacidad de Innovación Nacional de Furman, Porter y Stern (2002) que en esta sección se sistematizó.

\subsection{Estudio de los determinantes de la capacidad de innovación de las regiones españolas}

En este apartado se recoge el estudio de los determinantes de la capacidad de innovar de las regiones españolas, a partir del modelo de Furman, Porter y Stern (2002). Antes de presentar dicho estudio es importante comentar las siguientes cuestiones:

- En primer lugar, tal y como se ha comentado en la introducción del trabajo, en el caso español es especial- mente relevante el estudio regional. De este modo, la muestra del estudio de este trabajo la constituyen las 17 Comunidades Autónomas de España, que corresponden al nivel de desagregación NUTS II de acuerdo con la nomenclatura europea (Cuadro 3).

- En segundo lugar, es necesario detaIlar la forma en que el modelo de Furman, Porter y Stern (2002) se ha adaptado para el estudio regional, lo que supone una novedad frente a la literatura previa. De manera más precisa, dicha adaptación consiste en la utilización de las variables identificadas en el modelo, presentado en la sección previa de este trabajo, pero referidas en este caso al conjunto de regiones españolas. En concreto, debido a la disponibilidad de datos, no se han podido utilizar el total de variables utilizadas en la literatura previa presentada, y se toman las variables: PIBpc, población, personal científico técnico, gasto de las administraciones en I+D, gasto en educación secundaria y terciaria, gasto en I+D de las empresas, especialización productiva, y gasto en $\mathrm{I}+\mathrm{D}$ de las universidades. Estas coinciden con las utilizadas por Furman, Porter y Stern (2002) (modelo 4.4) y Gans y Stern

Cuadro 3

Muestra de regiones (2001-2005)

\begin{tabular}{llll}
\hline Andalucía & Cantabria & Extremadura & País Vasco \\
Aragón & Castilla-León & Galicia & Rioja \\
Asturias & Castilla la Mancha & Madrid & \\
Baleares & Cataluña & Murcia & \\
Canarias & Valencia & Navarra & \\
\hline
\end{tabular}

Fuente: Elaboración propia. 
Análisis de la capacidad de innovación regional: el caso español López-Fernández, María C.; Serrano-Bedia, Ana M. y García-Piqueres, Gema

(2003). Las variables han sido construidas en base a la revisión de la literatura, y en este sentido, el Cuadro 4 contiene la información sobre las variables utilizadas para cada dimensión, la descripción acerca de la manera en que cada una de ellas ha sido construida, y la fuente de datos utilizada en cada caso.

- En tercer lugar, el estudio empírico se ha llevado a cabo usando datos de diferentes encuestas desarrolladas por el Instituto Nacional de Estadística (INE) en el periodo 2001-2005, ambos inclusive. En términos más precisos, las fuentes de datos usadas han sido: la "Encuesta de Propiedad Industrial", las estadísticas de "Contabilidad Regional de España", las "Series de Población", la "Estadística de I+D" y la "Estadística del Gasto Público en Educación".

Una vez presentadas las cuestiones anteriores, a continuación se recogen los análisis realizados. En primer lugar, el estudio presenta las frecuencias y correlaciones entre las diferentes variables tomadas de la revisión de la literatura como determinantes de la capacidad de innovación. En cuanto al análisis de las correlaciones, si se presta atención a las correlaciones entre la variable dependiente y el resto de variables (independientes) (Tabla 1), la variable dependiente (PATENTES $(t+3)$ ), que mide la capacidad de innovación, es positiva y significativamente correlacionada con todas las variables del enfoque de la capacidad de innovación de Furman, Porter y Stern (2002), con la excepción de tres variables de especialización (ESPEC_AGRIC; ES-
PEC_ENERGIA; ESPEC_CONSTRU). Estos resultados sugieren que tanto la Calidad de la Infraestructura Común de la Innovación como el entorno específico de los clusters y la calidad de los vínculos, guardan una relación positiva y significativa con la capacidad de innovación regional. Asimismo, se analizan si los valores de las diferentes variables varían significativamente de unas regiones a otras. Para saber si dichas diferencias son estadísticamente significativas se han llevado a cabo test no paramétricos de KruskalWallis. En todos los casos las diferencias entre regiones resultan estadísticamente significativas al 0,01 , lo que sugiere la existencia de diferencias regionales en cuanto a sus actividades de innovación, y los principales factores en que éstas se apoyan. Estas diferencias regionales pueden venir determinadas tanto por las características específicas de cada región como por la diversidad de políticas de innovación dada la descentralización regional en algunas materias de esta índole para el caso español.

En segundo lugar, se aborda el estudio de los determinantes de la capacidad de innovación regional según el modelo de Furman, Porter y Stern (2002), adaptado al nivel regional, mediante análisis de regresión. Dada la tipología de datos que se utilizan en el estudio, que proporciona información de las variables para diferentes años (2001 a 2005), la aplicación de la metodología de datos de panel es posible. Esta metodología proporciona serias ventajas frente a las estimaciones de sección cruzada. La notación estándar de un modelo de datos de panel se representa como: 


$$
y_{i t}=\alpha_{i}+x_{i t} \beta+\varepsilon_{i t}
$$

donde i significa la i-ésima unidad transversal $(i=1, \ldots, N)$ y tel tiempot $(t=1, \ldots, T)$.

$x_{i t}$ es el valor de la variable para la unidad transversal i en t;

$\beta$ es un parámetro desconocido;

y $\varepsilon_{i t}$ es el término de error.

Esta metodología ofrece la posibilidad de controlar la heterogeneidad individual inobservable a través de la inclusión de un parámetro $\eta_{i}$ que contiene la información relativa a los efectos individuales o características específicas de cada unidad incluida en la muestra, características que son invariables en el tiempo.

No obstante, para saber si la estimación que procede es la de datos de panel o la del pool de datos o datos agrupados, se han llevado a cabo dos pruebas estadísticas. En primer lugar, se ha comparado la estimación del pool de datos con la estimación de datos de panel con efectos fijos (prueba F), y en segundo lugar con la estimación de datos de panel con efectos aleatorios (prueba de Breuch-Pagan o del Multiplicador de Lagrange). En este sentido tanto la prueba $\mathrm{F}$ (F-test= $9,48^{* * *}$ ) como la prueba de Breusch y $\mathrm{Pa}$ gan (Prueba de Breusch y Pagan: Chi-2= $36,38^{* * *}$ ) apuntan a que la metodología de datos de panel es la apropiada.

Otra cuestión fundamental relacionada con la metodología de datos de panel es la existencia o no de correlación entre la heterogeneidad inobservable $\left(\eta_{\mathrm{i}}\right)$ y los regresores $\left(\chi_{i t}\right)$. Si dicha correlación es cero, esto es que $\operatorname{Cov}\left(\chi_{\mathrm{it}}, \eta_{\mathrm{i}}\right)=0, \mathrm{t}=1$, $2, \ldots, \mathrm{T}$, entonces la estimación de efectos aleatorios es la adecuada. Si por el contrario, $\eta_{\text {i }}$ está correlacionada con $\chi_{i t}$, se debe realizar una estimación de efec- tos fijos (Wooldridge, 2002). Con el objetivo de conocer cual de las dos opciones es más apropiada para nuestro estudio se lleva a cabo un test de Hausman, resultando que la estimación oportuna es la de efectos aleatorios (Test de Hausman: Chi-2= 14,18).

Una vez que las pruebas anteriores indican que la metodología de datos de panel es la más apropiada, y dentro de ella, la estimación de efectos aleatorios, en base a la discusión de la literatura presentada en la sección anterior presentamos la siguiente ecuación que representa nuestro modelo empírico, que se estima en base a la metodología de datos de panel para la región i en el periodo de tiempo t:

$$
\begin{aligned}
& \text { LPATENTES }_{\mathrm{i}(\mathrm{t}+3)}=\alpha_{\mathrm{i}}+\beta_{1} \text { LPIBpc }_{\mathrm{it}}+\beta_{2} \\
& \mathrm{LPOB}_{i \mathrm{t}}+\beta_{3} \mathrm{EJC}_{-} \mathrm{C} \& \mathrm{I}_{\mathrm{it}}+\beta_{4} \\
& \text { LGASTO_ADMONES }_{\mathrm{it}}+\beta_{5} \text { TASA_EDUC }{ }_{\mathrm{it}}+ \\
& \beta_{6} \text { LGASTO_EMPRESAS }{ }_{\mathrm{it}}+\beta_{1} \text { ESPE_ENER- } \\
& \mathrm{GIA}_{\text {it }}+\beta_{8} \text { ESPE_CONSTRU }_{\text {it }}+\beta_{9} \\
& \text { ESPE_SERVI }_{\text {it }}+\beta_{10} \text { LGASTO_UNIV }_{i t}+\varepsilon_{\text {it }}
\end{aligned}
$$

Al igual que en trabajos previos, algunas de las variables se han tomado en logaritmos para realizar los análisis de regresión (PATENTES $(t+3)$; PIBpc; POB; GASTO_ADMONES; GASTO_EMPRESAS; GASTO_UNIV). Asimismo, siguiendo a autores previos (Furman, Porter y Stern, 2002; Furman y Hayes, 2004; Hu y Mathews, 2005; Hu y Mathews, 2008; Krammer, 2009) se ha optado por una metodología en la que las variables se introducen en pasos. De este modo, se han desarrollado cuatro modelos denominados Función de Producción de Ideas, Infraestructura Común de la Innovación, Infraestructura y Entorno de los Cluster y Capacidad de Innovación Regional. El 
Análisis de la capacidad de innovación regional: el caso español López-Fernández, María C.; Serrano-Bedia, Ana M. y García-Piqueres, Gema
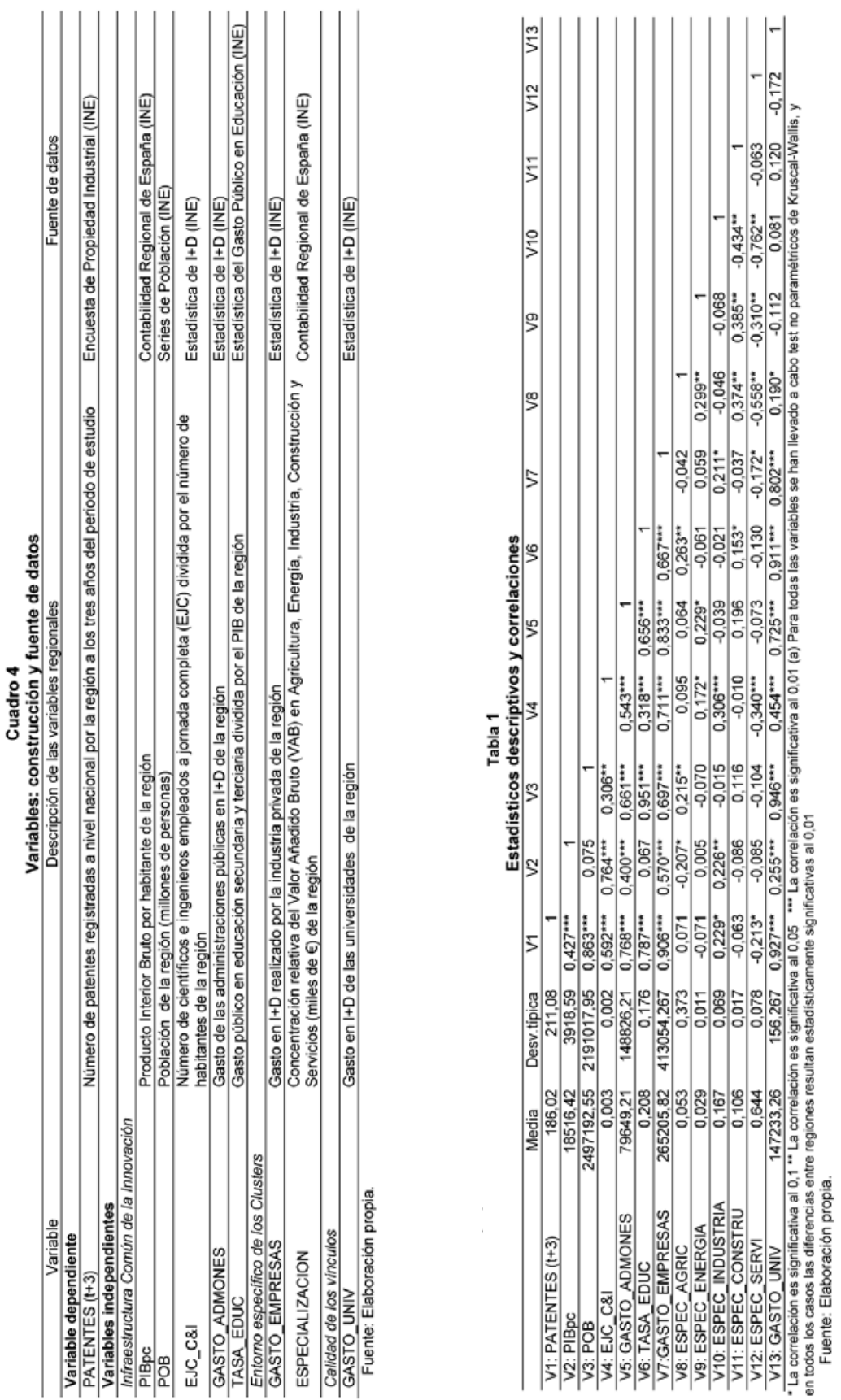
primero de los modelos consiste en la especificación relativa a la Función de Producción de Ideas de los modelos de crecimiento endógeno presentados anteriormente (Romer, 1986; 1990) que relaciona el stock de conocimiento acumulados y el personal dedicado a I+D como principales motores de la generación de innovaciones. Por tanto este modelo contiene las variables LPIBpc, LPOB y EJC_C\&I. El segundo de los modelos del estudio empírico agrupa el conjunto de variables determinantes de la capacidad de innovación pertenecientes a la dimensión Infraestructura Común de la Innovación del modelo de Furman, Porter y Stern (2002) (ver Cuadro 4). El tercer modelo consiste en las variables relativas a las dimensiones Infraestructura Común de la Innovación y Entorno Específico de los Clusters del modelo de Furman, Porter y Stern (2002) (ver Cuadro 4). Por último, el cuarto modelo representa el enfoque de Furman, Porter y Stern (2002) de manera global, por incluir las variables relativas a las tres dimensiones identificadas por dichos autores como determinantes de la capacidad de innovación (ver Cuadro 4).

La Tabla 2 recoge los resultados de la estimación de datos de panel con efectos aleatorios, que se comentan de forma detallada a continuación. Comenzando con el modelo de la "Función de Producción de Ideas", el modelo presenta un buen ajuste por cuanto que el valor de la Chi-2 es alto y estadísticamente significativo al $1 \%$. Comenzando con la variable LPIBpc, se obtiene un efecto significativo y positivo, resultado que esta en la misma línea que la mayor parte de la evidencia empírica previa (Cuadro 2 y Figura 2). En cuanto a la variable LPOB el efecto es po- sitivo y significativo al igual que algunos trabajos previos, si bien los resultados de éstos no eran concluyentes (Cuadro 2 y Figura 2). Respecto de la variable EJC C\&I, los resultados obtenidos indican un efecto no significativo de esta variable sobre la capacidad de innovación regional.

El segundo de los modelos, "Infraestructura Común de la Innovación", ajusta asimismo bien, con significatividad del $1 \%$. Por un lado, en este modelo los resultados respecto de las variables LPIBpc, LPOB y EJC C\&I coinciden con los del modelo anterior. Respecto a LGASTO_ADMONES y TASA_EDUC, los resultados obtenidos indican un efecto no significativo de ambas variables sobre la capacidad de innovar de las regiones.

El tercer modelo incluye el conjunto de variables relativas a la "Calidad de la Infraestructura Común de la Innovación" por un lado, y al "Entorno Específico de los Clusters" por otro, de acuerdo con el modelo de Furman, Porter y Stern (2002). Al igual que los dos modelos anteriores, presenta un buen ajuste significativo al $1 \%$. Las variables LPIBpc, LPOB mantienen la significatividad como en modelos anteriores y a ellas se suma la variable LGASTO_ADMONES, que mantiene el signo positivo y pasa a ser estadísticamente significativa, resultado coincidente con la mayoría de la evidencia empírica previa (cuadro 2 y figura 2). Por otro lado, y respecto de las variables del Entorno Especifico de los Clusters, los resultados indican un efecto no significativo de la variable LGASTO_EMPRESAS sobre la capacidad de innovación regional. Por lo que a la especialización productiva se refiere, solo la variable ESPEC_INDUSTRIA, tiene efectos estadísticamente sig- 
Análisis de la capacidad de innovación regional: el caso español

López-Fernández, María C.; Serrano-Bedia, Ana M. y García-Piqueres, Gema

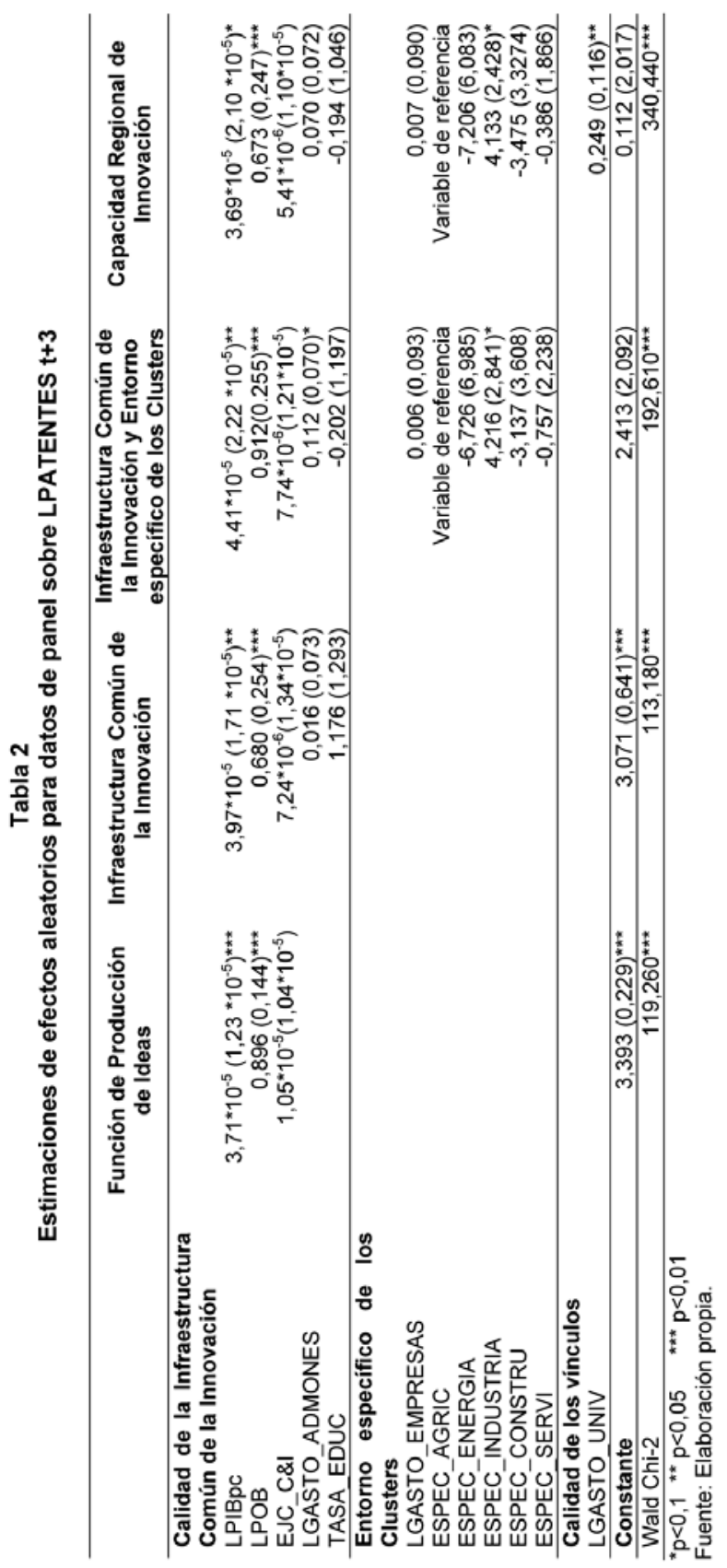


nificativos sobre la capacidad de innovación regional. En este caso, los resultados indican que las regiones más especializadas en este tipo de actividad tienen mayor capacidad de innovación, un resultado que se explica entre otras razones por el tipo de variable dependiente utilizada (patentes). En último lugar, el modelo "Capacidad Regional de Innovación" es el que presenta el mejor ajuste de los tres modelos, con el valor más alto de la Chi-2, significativo al $1 \%$. Los resultados de este modelo son coincidentes con los del modelo anterior con la excepción de la variable LGASTO_ADMONES, que en este caso pierde la significatividad. Por otro lado, en este modelo se ha incluido una variable respecto al anterior: LGASTO_UNIV, confirmando estadísticamente los análisis su efecto positivo sobre la capacidad de innovación regional, un resultado coincidente con la mayor parte de la evidencia empírica previa (Cuadro 2 y Figura 4).

\section{Conclusiones}

El objetivo de este trabajo ha sido doble: Por un lado, comprobar si el modelo de capacidad de innovación nacional desarrollado por Furman, Porter y Stern (2002) se puede adaptar para el nivel de análisis regional. Por otro, el estudio de los determinantes de la capacidad de innovación regional en España, usando dicho modelo.

Las conclusiones que se desprenden de este estudio se han clasificado en dos grandes grupos: 1) implicaciones teóricas, e 2) implicaciones de gerencia. Por último, en este apartado se recogen las limitaciones y posibles futuras investigaciones derivadas de ese trabajo.

Las principales implicaciones teóricas del trabajo presentado guardan relación con la elección del modelo teórico utilizado como referencia, el elaborado por Furman, Porter y Stern (2002), y su pertinencia o adecuación para la realización de análisis a nivel regional. En relación con este aspecto es preciso señalar que a la vista de los ajustes de los modelos desarrollados en el estudio empírico ("Función de Producción de Ideas"; "Infraestructura Común de la Innovación”; y "Infraestructura Común de la Innovación y Entorno Especifico de los Cluster"; "Capacidad Regional de Innovación") se pone de manifiesto, en primer lugar, la pertinencia de utilizar el marco de la Capacidad de Innovación (Furman, Porter y Stern, 2002) para el caso regional, lo que viene a dar robustez a los resultados de los análisis de las correlaciones que iban en esta misma línea. En segundo lugar, el hecho de que sea el cuarto modelo propuesto, que agrupa las tres dimensiones de determinantes de la capacidad de innovar propuestas por Furman, Porter y Stern (2002), el que mejor ajuste presenta refleja la importancia de considerar los tres tipos de variables identificadas en dicho modelo.

Por otro lado, los resultados del estudio empírico, que concuerdan en gran medida con la literatura previa aplicada a nivel nacional, muestran que para el caso de las regiones españolas los factores relacionados con la infraestructura común de la innovación y la calidad de los vínculos se configuran como principales motores de su capacidad de innovación. De 
Análisis de la capacidad de innovación regional: el caso español

López-Fernández, María C.; Serrano-Bedia, Ana M. y García-Piqueres, Gema

una forma más precisa, si se analizan los componentes de cada uno de los tres elementos identificados en el modelo, dentro de la infraestructura común de la innovación son el PIBpc, la población y el gasto en I+D de las administraciones públicas, los factores significativos en la determinación de la capacidad de innovación regional. Estos resultados son similares a los obtenidos en trabajos empíricos previos respecto del PIBpc, y el gasto en I+D, mientras que para el caso de la población, en que la evidencia empírica previa no era concluyente nuestros resultados confirman su efecto positivo sobre la capacidad de innovación regional. Respecto de la calidad de los vínculos, se confirma la significatividad del gasto de las universidades en I+D sobre la capacidad de innovación regional, al igual que en la literatura empírica previa.

Si se comparan estos resultados con los valores de las variables a nivel regional, se comprueba que son las regiones de Cataluña, Madrid, Valencia y Andalucía las que presentan los valores más elevados en las variables que han resultado significativas en el estudio. En términos más precisos, las cuatro regiones alcanzan las primeras posiciones en términos de población, gasto en $I+D$ de las administraciones públicas y las universidades, si bien Cataluña presenta una mayor especialización en la industria y el resto en los servicios. Por lo tanto, se puede concluir que, con independencia de su modelo de especialización productivo, estas regiones cuentan con los recursos clave, humanos y financieros, para potenciar su capacidad de innovación. Este resultado apuntaría a la necesidad de que exista un umbral mínimo de recursos invertidos en $\mathrm{I}+\mathrm{D}$ en las regiones para poder obtener resultados de sus políticas de innovación. Además, en torno a la capacidad de innovación, los datos muestran que son precisamente estas cuatro regiones las que presentan una mayor capacidad de innovación en términos de generación de patentes, con valores muy por encima de la media para el período estudiado. En concreto, frente a una producción media para el conjunto de regiones de 152 patentes, Cataluña, Madrid, Valencia y Andalucía produjeron una media por año de 742, 610, 375 y 354 patentes respectivamente. Lo que en términos absolutos supuso un $66 \%$ de la producción total de patentes de las 17 regiones.

En cuanto a las implicaciones de este estudio para la gerencia, a continuación se identifican una serie de ellas a nivel de responsables de administraciones. En este caso, el conocimiento de los factores que determinan la capacidad de innovación regional puede resultar de interés para mejorar el diseño de políticas de apoyo a la innovación en este ámbito, que en el contexto español se materializan principalmente mediante tres tipos de programas de ayudas (Sánchez Bueno, 2008): Programas Marco (ámbito europeo), Planes Nacionales de I+D+i (ámbito nacional) y Programas Regionales de I+D+i (ámbito regional).

La principal recomendación que se puede extraer de los resultados de este estudio empírico es que la infraestructura común para la innovación y los vínculos entre ésta y los clusters empresariales son importantes dinamizadores de la innovación regional y deben, por lo tanto, ser tenidas en cuenta a la hora del diseño 
de políticas. Sin embargo, el papel de las empresas parece más limitado, lo que sugiere cierta debilidad en cuanto a la generación de innovaciones por parte de las empresas españolas. El perfil de pequeña y mediana empresa con gran presencia en el sector servicios hace que el tejido empresarial no tenga una gran proactividad en términos de innovación, tradicionalmente más ligada a la empresa grande e industrial o manufacturera. En este sentido, una sugerencia a los responsables del diseño de políticas de innovación regionales es incorporar programas de actuación que complementen los desarrollados a nivel nacional y europeo. Así, por ejemplo, programas dirigidos a incorporar a investigadores de reconocido prestigio internacional en centros regionales de investigación, a fomentar la colaboración entre empresas y universidades y centros públicos de investigación de la región, a potenciar la actividad industrial como área de desarrollo e innovación tecnológica o a apoyar la investigación básica de las universidades y su transferencia al tejido empresarial podrían ser medidas útiles para potenciar la capacidad innovadora regional.

Por otra parte, es importante mencionar como la principal limitación los datos disponibles, que ha condicionado la forma en que se han construido algunas de las variables.

Asimismo, en términos de futuras líneas de investigación, en primer lugar podría resultar relevante ampliar el estudio a todas las regiones europeas a nivel de desagregación NUTS II. En segundo lugar, los resultados del estudio sugieren el interés de profundizar en el análisis de las políticas de innovación que desarrollan las regiones con mayor capacidad innovadora, con el objetivo de conocerlas de manera pormenorizada y explorar sus posibilidades de aplicación en otros contextos regionales.

\section{Referencias bibliográficas}

Abramovitz, Moses (1956). Resource and Output Trends in the United States Since 1870, American Economic Review, Vol. 46, pp. 5-23.

Aghion, Philippe y Howitt, Peter (1992). A Model of Growth Through Creative Destruction, Econometrica, Vol. 60, pp. 323-351.

Arrow, Kenneth. J. (1962). The Ecomomic Implications of Learning by Doing, Review of Economic Studies, Vol. 29, pp. 155-173.

Batlle Rois-Méndez, Francisco e Inciarte Osorio, Bárbara (2009). Gerencia del conocimiento en empresas cerveceras. Estudio de un caso, Revista Venezolana de Gerencia, Vol. 14, No. 54, pp. 42-54.

Baumert, Thomas y Heijs, Joost (2002). Los determinantes de la capacidad innovadora regional: una aproximación econométrica al caso español. Recopilación de estudios y primeros resultados, Documento de Trabajo No. 33, Instituto de Análisis Industrial y Financiero, Universidad Complutense, Madrid (www.ucm.es/BUCM/cee/iaif).

Buesa, Mikel, Heijs, Joost, Martínez Pellitero, Mónica y Baumert, Thomas (2006). Regional systems of innovation and the knowledge production function: the Spanish case, Technovation, Vol. 26, pp. 463-472.

Chatterton, Paul y Goddard, John (2000). The Response of Higher Education Institutions to Regional Needs, European Journal of Education, No. 35, Vol. 4, pp. 475-496. 
Análisis de la capacidad de innovación regional: el caso español

López-Fernández, María C.; Serrano-Bedia, Ana M. y García-Piqueres, Gema

Comisión Europea (2001). Second report on economics and social cohesion, Comisión Europea, http://ec.europa.eu/regional_policy/sources/docoffic/official/reports/contentpdf_en.htm.

Coronado, Daniel y Acosta, Manuel (1999). Innovación Tecnológica y Desarrollo Regional, Información Comercial Española Octubre, Vol. 781, pp. 103-116.

COTEC (2004). Libro Blanco 2004- El Sistema Español de Innovación: Situación en 2004, COTEC, Madrid.

Denison, Edward F. (1962). United States Economic Growth, Journal of Business, Vol. 35, pp. 109-121.

Denison, Edward F. (1967). Why Growth Rates Differ: Post-War Experience in Nine Western Countries, Brookings Institution, Washington, D. C.

Dosi, Giovanni (Ed.) (1988). Technical change and economic theory, Pinter Publishers, London, UK.

Edquist, Charles (1997). Systems of Innovation: Technologies, institutions and organization, Pinter Publishers, London, UK.

Estrada Bárcenas, Roberto, García Pérez de Lema, Domingo y Sánchez Trejo, Victor Gabriel (2009). Factores determinantes del éxito competitivo de la Pyme: Estudio empírico en México, Revista Venezolana de Gerencia, Vol. 14, No. 46, pp. 169-182.

Faber, Jan y Hesen, Anneloes Barbara (2004). Innovation capabilities of European nations Cross-national analyses of patens and sales of product innovations, Research Policy, Vol. 33, pp. 193-207.

Fagerber, Jan (1987). A technology gap approach to why growth rates differ, Research Policy, Vol. 16, pp. 15711583.
Fagerberg, Jan (1988). Why growth rates differ, G. Dosi et al. (Eds.), Technical Change and Economic Theory, Pinter, London, pp. 432-457.

Fagerberg, Jan (1991). Innovation, catching up and growth, Technology and Productivity: the Challenge for Economic Policy, Paris: OECD, pp. 37-46.

Fagerberg, Jan, Mowey, David C. y Nelson, Richard R. (2006) (Eds.). The Oxford Handbook of Innovation, Oxford University Press, Oxford.

Fernández Estrada, Oscar, Roibás, David y Triana, Juan (2007). Estudio sobre los factores determinantes de la capacidad nacional innovativa en América Latina y El Caribe, Trabajo para Suficiencia Investigativa, Universidad de la Habana-Universidad de Oviedo.

Fernández de Lucio, Ignacio, Conesa, Fernando, Garea, M., Castro, Elena, Gutiérrez, Antonio (1996). Estructuras de Interfaz en el Sistema Español de Innovación. Su papel en la Difusión de Tecnología, CTT, Universidad Politécnica de Valencia.

Fernández de Lucio, Ignacio (2001). Las debilidades y fortalezas del sistema valenciano de innovación, en Olazarán, Mikel, Gómez Uranga, Mikel (Eds.), Sistemas Regionales de Innovación, País Vasco, pp. 251-278.

Fernández de Lucio, Ignacio, Castro Martínez, Elena y Zabala Iturriagagoitia, Jon Mikel (2007). Estrategias regionales de innovación: el caso de regiones europeas periféricas en Vence Deza, Xavier (Ed.), Crecimiento y políticas de innovación. Nuevas tendencias y experiencia comparadas, Ediciones Pirámide, Madrid, pp. 157-189.

Freeman, Christopher (1987). Technology Policy and Economic Performance. Lessons from Japan, Printer, London. 
Furman, Jeffrey L. y Hayes, Richard (2004). Catching up or standing still? National innovative productivity among 'follower' countries, 1978-1999, Research Policy, Vol. 33, pp. 13291354.

Furman, Jeffrey L., Porter, Mihael E. y Stern, Scott (2002). The determinants of national innovative capacity, Research Policy, Vol.31, pp. 899-933.

Gans, Joshua y Stern, Scott (2003). Assesing Australia's innovative capacity in the $21^{\text {st }}$ century, Working Paper 2003-16, Melbourne Business School.

González Ramos, Ana. M. y González de la Fe, Teresa (2009). Los actores de los sistemas de I+D+i en las regiones periféricas, Papers, Vol. 91, pp. 65-80.

Gregersen, Birgitte y Johnson, Björn (1997). Learning economies, innovation systems and European integration, Regional Studies, Vol. 31, pp. 479-490.

Grossman, Gene M. y Helpman, Elhanan (1991). Innovation and Growth in the Global Economy, Cambridge, MA: MIT Press.

Heijs, Joost, Buesa, Mikel y Baumert, Thomas (2007). Sistemas nacionales de innovación: conceptos, perspectivas y desafíos, en: Buesa, Mikel, Heijs, Joost, Asheim, Björn, Baumert, Thomas, Navarro, Mikel y Martínez Pellitero, Mónica. Sistemas regionales de innovación: nuevas formas de análisis y medición, Fundación de Cajas de Ahorros (FUNCAS), Madrid, pp. 17-65.

$\mathrm{Hu}$, Mei Chih y Mathews, John A. (2005). National innovative capacity in East Asia, Research Policy, Vol. 34, pp. 13221349.

$\mathrm{Hu}$, Mei Chih y Mathews, John A. (2008). China's national innovative capacity, Research Policy, Vol. 37, pp. 1465-1479.

Jones, Charles (1988). Introduction to the Economic Growth, WW Norton\&Co., New York.
Krammer, Sorin M.S. (2009). Drivers of national innovation in transition: evidence from a panel of Eastern European countries, Research Policy, Vol. 38, pp. 845-860.

Lucas, Robert E. (1988). On the mechanisms of the Economic Development, Journal of Monetary Economics, Vol. 22, pp. 435-444.

Lundvall, Bengt-Åke (1985). Product innovation and User-Producer Interaction, Aalborg University Press, Aalborg.

Lundvall, Bengt-Åke (1992). National Systems of Innovation: Towards a Theory of Innovation and Interactive Learning, Printer, London.

Meyer-Kramer, Frieder (2001). Industrial innovation and sustainability-conflicts and coherence, Archibugi, Daniele y Lundvall Bengt-Åke (Eds.), The globalizing economy, Oxford University Press, Oxford, pp 177-194.

Mitxeo Grajirena, Jone, Idigoras Gamboa, Idoia y Vicente Molina, Azucena (2004). Los clusters como fuente de competitividad: el caso de la Comunidad Autónoma del País Vasco, Cuadernos de Gestión, Vol. 4, No. 1, pp. 55-67.

Mowery, David C. (1984). Firm structure, government policy, and the organization of industrial research: Great Britain and the United States, 1900-1950, Business History Review, Vol. 58, pp. 504-531.

Nelson, Richard R. (1993) (Ed.). National Innovation System: A comparative analysis, Oxford University Press, New York.

Nelson, Richard. R. y Winter, Sidney G. (1982). An evolutionary theory of economic change, Harvard University Press, Cambridge.

Niosi, Jorge (1991). Canada's National System of Innovation, Science and Public Policy, Vol. 18, No. 4, pp. 83-92. 
Análisis de la capacidad de innovación regional: el caso español

López-Fernández, María C.; Serrano-Bedia, Ana M. y García-Piqueres, Gema

Ojeda Gómez, Julieta (2007). Ventaja competitiva: el reto de las PyME en la industria del calzado, Revista Venezolana de Gerencia, Vol. 12, No. 40, pp. 513-533.

Park, Sang-Chul y Lee, Seong-Keun (2005). The national and regional innovation systems in Findland: from the path dependency to the path creation approach, Al \& Society, Vol. 19 No. 2, pp. $180-195$.

Peña Cedillo, Jesús y Flores Urbáez, Matilde (2006). Evaluación de las agendas de investigación e innovación en Venezuela, Revista Venezolana de Gerencia, Vol. 11, No. 33, pp. 29-46.

Porter, Michael E. (1990). The Competitive Advantage of Nations, Free Press, New York.

Porter, Michael E., Furman, Jeffrey L. y Stern, Scott (2001). Los factores impulsores de la capacidad innovadora nacional: implicaciones para España y América Latina, Claves de la Economía Mundial, ICEX: Madrid, pp. 78-88.

Riddel, Mary y Schwer, R. Keith (2003). Regional innovative capacity with endogenous employment: empirical evidence from the U.S, The Review of Regional Studies, Vol. 33 No. 1, pp. 73-84.

Romer, Paul M. (1986). Increasing Returns and Long Run Growth, Journal of Political Economy, Vol. 94, pp. 1002-1037.

Romer, Paul M. (1987). Growth based on increasing returns due to specialization, American Economic Review, Vol. 77, pp. 56-62.
Romer, Paul M. (1990). Endogenous technological change, Journal of Political Economy, Vol. 98, S71-S102.

Sánchez Bueno, María José (2008). El proceso innovador y tecnológico. Estrategias y apoyo público, Netbiblo, La Coruña.

Solow, Robert M. (1956). A contribution to the theory of economic growth, The Quaterly Journal of Economics, Vol. 70 No. 1, pp. 65-94.

Solow, Robert M. (1957). Technical change and the aggregate production function, The Review of Economics and Statistics, Vol. 39 No. 3, pp. 312-320.

Varsakelis, Nikos C. (2006). Education, political institutions and innovative activity: a cross-country empirical investigation, Research Policy, Vol. 35, pp. 1083-1090.

Verspagen, Bart (2000). Economic growth and technological change: an evolutionary interpretation, OECD STI Working Papers, NO. JT00101276.

Wiehler, Frank y Stumm, Thomas (2005). The powers of regional and local authorities and their role in the European Union, European Planning Studies, Vol. 3, pp. 227-250.

Wooldridge, Jeffrey M. (2002), Econometric analysis of Cross-section and $\mathrm{Pa}$ nel-data, MIT Press, Cambridge, MA. 